\title{
Dynamic Folding Pathway Models of the Villin Headpiece Subdomain (HP-36) Structure
}

\author{
IN-HO LEE, ${ }^{1}$ SEUNG-YEON KIM, ${ }^{2}$ JOOYOUNG LEE ${ }^{3}$ \\ ${ }^{1}$ Korea Research Institute of Standards and Science, Daejon 305-600, Korea \\ ${ }^{2}$ School of Liberal Arts and Sciences, Chungju National University, Chungju 380-702, Korea \\ ${ }^{3}$ Korea Institute for Advanced Study, Seoul 130-722, Korea \\ Received 1 August 2008; Revised 22 January 2009; Accepted 4 March 2009 \\ DOI 10.1002/jcc.21288 \\ Published online 1 May 2009 in Wiley InterScience (www.interscience.wiley.com).
}

\begin{abstract}
We have investigated the folding pathway of the 36-residue villin headpiece subdomain (HP-36) by actionderived molecular dynamics simulations. The folding is initiated by hydrophobic collapse, after which the concurrent formation of full tertiary structure and $\alpha$-helical secondary structure is observed. The collapse is observed to be associated with a couple of specific native contacts contrary to the conventional nonspecific hydrophobic collapse model. Stable secondary structure formation after the collapse suggests that the folding of HP-36 follows neither the framework model nor the diffusion-collision model. The C-terminal helix forms first, followed by the N-terminal helix positioned in its native orientation. The short middle helix is shown to form last. Signs for multiple folding pathways are also observed.
\end{abstract}

(C) 2009 Wiley Periodicals, Inc. J Comput Chem 31: 57-65, 2010

Key words: protein folding; molecular dynamics; pathway

\section{Introduction}

Protein folding problem is one of the important problems of the contemporary biology. ${ }^{1}$ The mechanism that leads to the folding of proteins is not yet clarified and despite much progress in the field, the issue remains as one of the major unsolved problems. Understanding the protein folding is particularly important in this postgenomic era. In the last decade, various experimental techniques including $\mathrm{X}$ ray and NMR spectroscopies are developed to illustrate glimpses of folding pathways. These techniques now allow us to investigate fast folding events in the time scale of microseconds rather than the usual milliseconds. Nevertheless, the current spatial/temporal resolution is not yet sufficiently detailed/fast enough to investigate early folding events directly at the atomic scale. ${ }^{2}$

Computer simulation is a useful tool to study kinetic processes and thermodynamic stabilities of protein folding. ${ }^{3-12}$ The most direct atomistic simulation technique is the conventional molecular dynamics (MD) method, ${ }^{13}$ in which atomic motions are calculated by integrating the classical equations of motion for a given potential-energy function. Complementary to experiments, the MD simulation can provide time-dependent description of a protein system and, in principle, it can solve the protein folding problem. However, direct atomistic protein folding simulations are severely limited by the short time scale accessible with the current computational methods and resources. ${ }^{3}$ The size and the type of a protein fold that can be treated by folding simulations are quite limited in practice. However, cumulative understanding arising from various computational folding studies of small proteins cannot be underestimated. ${ }^{3,5,8-10}$ A set of small proteins provides a challenge for testing/identifying the protein folding mechanism.

Because of the stochastic nature of the folding process associated with crossing a large free energy barrier between the initial unfolded and the final folded conformations, even fast folding events of a small protein (domain) require long simulation time (longer than a nano-second). This directly limits the application of standard MD simulations to proteins. ${ }^{3}$ For this reason, we have not, yet, witnessed the folding of a protein over 100 amino acid residues by direct MD simulations. This is due to the lack of not only computational resources but also proper computational methodologies. Even with a small protein, to alleviate the situation, folding/unfolding MD simulations are often carried out at nonphysiologically high temperatures. ${ }^{7}$ Another approach is to use many separate short-time MD simulations assuming exponential kinetics of folding events. ${ }^{8}$

Recently, we proposed that the action-derived molecular dynamics (ADMD) $)^{14,15}$ method can be useful for the study of rare events, especially for protein folding, and demonstrated that much insights can be obtained through ADMD studies. ${ }^{16}$ Because ADMD transforms the initial value problem of the conventional MD into a boundary value problem, it is particularly well suited for the study of

Correspondence to: J. Lee; e-mail: jlee@kias.re.kr

Contract/grant sponsor: Creative Research Initiatives (Center for In Silico Protein Science) of MEST/KOSEF 
rare events associated with large potential-energy barriers. For given initial and final states, ADMD can provide low-potential-energy pathways between them.

ADMD has been successfully applied to folding studies of an $\alpha$ helix (acetyl-(Ala) $10-N$-methyl amide) and a $\beta$-hairpin (residues 41 56 of protein $\mathrm{G}) .{ }^{16}$ The calculated folding pathways were consistent with experimental data, reproducing correct sequential formation of native hydrogen bonds. Starting from extended conformations, both the $\alpha$ helix and the $\beta$-hairpin were found to form by cooperative folding.

In this article, we investigate the folding pathway of $\mathrm{HP}-36^{17}$ at atomic details by using ADMD and parallel computation. HP-36 is a 36-residue domain from the villin headpiece with three $\alpha$-helices (pdb-code is 1VII with amino acid sequence of MLSDEDFKAVFGMTRSAFAN-LPLWKQQNLK-KEKGLF), and it is known to fold cooperatively into its compact native state. ${ }^{18-21}$ With a wellpacked hydrophobic core, HP-36 is thermodynamically stable under its folding condition. Because of its ultrafast folding rate and many mutation studies, HP-36 has served as a popular target for many folding studies both by theory ${ }^{3,4,10,11,22}$ and experiment. ${ }^{17-21,23}$ However, the nature of the HP-36 folding dynamics still remains to be clarified due to the lack of its microscopic information from experiments. Successful microscopic folding study of HP-36 will help us to understand the general folding principle including the relationship between the secondary structure establishment and the formation of final tertiary contacts.

Although it is not the direct subject of this work, we would like to mention very recent MD studies on variants of the HP-36. The variants are HP-35 where the first amino acid $\mathrm{M}$ is missing from HP-36 and HP-35_NleNle where two lysines at the 24-th and 29th positions are replaced by norleucines to expedite the folding. Lei and Duan ${ }^{24}$ obtained folding trajectories of HP-35 through MD simulations, starting from the extended structure. The $\mathrm{C}^{\alpha}$ root-meansquare deviation (RMSD) between the X-ray crystal structure and the closest structure generated fell below $1.0 \AA$.

In addition, they found that residues 13-31 form prefolded helices which, once formed, remain throughout the folding trajectory. The entire folding is obtained via docking of the N-terminal helix (residues 2-11) to the prefolded helices. In a similar MD study on HP-35_NleNle, Ensign et al. ${ }^{25}$ showed that the rate of relaxation to the native state depends on the starting structure. These computational studies ${ }^{24,25}$ indicate that the fast folding of villin headpieces is due to the formation of stable helices (residues 13-31). Kubelka et al. ${ }^{21}$ investigated the structure and folding kinetics of HP-35 with the nanosecond laser T-jump technique. They found that the free energy barrier for HP-35 folding is rather small $(\sim 1 \mathrm{kcal} / \mathrm{mol})$, which suggests that the engineered HP-35 may serve as a more ideal protein system for kinetic studies.

\section{Methods}

The goal of this study is to investigate the protein folding mechanism of HP-36 by providing its sequential conformational changes from the initial extended structure to its final native structure, at atomic details. The time interval between successive conformational changes is set to be short enough (in the order of tens of femtoseconds) to describe the folding event in structural continuity, but long enough so that not-so-important fast vibrational modes are properly averaged out. Pathways from this study would correspond to average pathways with low potential-energy profile between two given states.

The advantages of the ADMD over the conventional MD method are, in ADMD, (i) no reaction coordinates need to be assigned prior to simulation, (ii) no arbitrary criterion to define the folded native structure is required, (iii) it is not necessary to assign initial velocities since the ADMD formalism utilizes only the coordinate information, and (iv) the simulation can be easily adopted for straightforward parallel computation. However, as in all the other studies, the simulation results depend on the accuracy/relevance of the force field used. We assume that low-potential-energy pathways generated in this study represent most relevant pathways allowed by the force field. Folding simulations are performed assuming no prior knowledge on the structural variation other than dictated by the force field.

At the beginning of each ADMD simulation, a set of random numbers is generated to construct a trial atomic trajectory for each atom, connecting the initial and the final conformations provided. We relate trajectories with low-potential-energy barriers as probable transition pathways. By applying the least action principle, the Newtonian dynamics formulation is now transformed into a boundary value problem to generate classical low-potential-energy trajectories bridging two given structures. An appealing feature of ADMD is that its trajectory globally follows a Newtonian trajectory according to the equations of motion. ${ }^{14,15,26}$

To start ADMD simulations, the initial and the final coordinates of the all constituting atoms should be provided. For HP-36, the total number of atoms is $N=596$. In this work, the final conformation is prepared by applying local energy minimization to the native structure of HP-36 obtained by NMR (pdb-code: 1 VII). ${ }^{17}$ The choice for the initial conformation is less obvious, and we used a local-energy-minimized structure generated from the fully extended conformation, as shown in Figure 1. The potential-energy difference between the initial and final conformations is measured to be 181.26 $\mathrm{kcal} / \mathrm{mol}$.

We discretized the whole atomic trajectory into $P=2000$ steps with the successive time increment of $\Delta=36.28 \mathrm{fs}$, the total simulation time being $\tau=P \Delta=72.56 \mathrm{ps}$. The path $\left\{\mathbf{q}_{j}\right\}$ is a collection of sequential structural frames with fixed initial $\mathbf{q}_{0}$ and final $\mathbf{q}_{P}$. The discretized action can be written as

$$
S=\sum_{j=0}^{P-1} L_{j}\left(\left\{\mathbf{q}_{j}\right\}\right) \Delta,
$$

where the discretized Lagrangian of the $j$ th temporal frame is defined as

$$
L_{j}=\sum_{I=1}^{N} \frac{m_{I}}{2 \Delta^{2}}\left(\mathbf{q}_{I, j}-\mathbf{q}_{I, j+1}\right)^{2}-V\left(\left\{\mathbf{q}_{j}\right\}\right) .
$$

Here, the first term is the kinetic energy, and $V$ is the potential energy. $N$ is the total number of atoms, $m_{I}$ is the mass of the $I$ th atom, and $\mathbf{q}_{I, j}$ is the position vector of the $I$ th atom at the $j$ th frame. 
The stationarity condition $\delta S=0$ leads to a set of linear equations. However, discretized pathways generated from the minimization of eq. (1) do not satisfy total energy conservation as discussed in the work of Passerone and Parrinello. ${ }^{14}$ That is, accurate Verlet trajectories ${ }^{27}$ are not guaranteed because the action of eq. (1) is not bounded. Passerone and Parrinello ${ }^{14}$ suggested adding a constraint term to eq. (1) to ensure the total energy conservation from pathways. The modified action is now

$$
\Theta\left(\left\{\mathbf{q}_{j}\right\} ; E\right)=S+\mu_{E} \sum_{j=0}^{P-1}\left(E_{j}-E\right)^{2},
$$

where $E$ is the target total energy value to impose on the system, $\mu_{E}$ is an arbitrary large constant, and $E_{j}$ is the total energy at the $j$ th frame defined as

$$
E_{j}=\sum_{I=1}^{N} \frac{m_{I}}{2 \Delta^{2}}\left(\mathbf{q}_{I, j}-\mathbf{q}_{I, j+1}\right)^{2}+V\left(\left\{\mathbf{q}_{j}\right\}\right)
$$

Quality of pathways is shown to improve further ${ }^{15}$ by introducing an additional dynamic restraint. Now the action to optimize becomes

$\Phi\left(\left\{\mathbf{q}_{j}\right\} ; E, T\right)=S+\mu_{E} \sum_{j=0}^{P-1}\left(E_{j}-E\right)^{2}+\mu_{K} \sum_{I=1}^{N}\left(\left\langle K_{I}\right\rangle-\frac{3 k_{B} T}{2}\right)^{2}$,

where $\left\langle K_{I}\right\rangle$ is the average kinetic energy of the Ith atom along the trajectory. ${ }^{15}$ Fictitious temperature $T$ controls the kinetic energy of the system, $\mu_{K}$ is an arbitrary large constant, and $k_{B}$ is the Boltzmann's constant.

It should be noted that $T$ used in this work does not correspond to the physical temperature. $T$ is only a parameter introduced to improve the quality of pathway by reducing the value of OnsagerMachlup action. ${ }^{14,15,26}$ A smaller value of Onsager-Machlup action corresponds to a more Verlet-like trajectory. In this work, we have optimized the eq. (5) to generate ADMD pathways with $3 N(P-1)=3 \times 596 \times 1999=3,574,212$ degrees of freedom. All atoms are treated as point particles with atomic masses according to their atom types $(\mathrm{H}, \mathrm{C}, \mathrm{N}, \mathrm{O}$, and $\mathrm{S})$. It should be noted that no artificial constraints are imposed on the covalent bond lengths and angles other than that they are subject to the force field used. We used the AMBER $94^{28}$ all-atom force field with GB/SA solvation model $^{29}$ as implemented in the TINKER package. ${ }^{30}$

For the rigorous minimization of the action defined in eq. (5), one should consider to apply a global optimization method such as the simulated annealing ${ }^{31}$ or the conformational space annealing. ${ }^{32,33}$ However, because the execution of even a local minimization takes a significant amount of computational resources, we decided to perform 20 separate local minimizations. For the local minimization, a multigrid method ${ }^{34}$ is used where the number of conformations $(P)$, initially as small as 20 , continues to grow to reach 2000 at the final stage. For a given $P$ we used the quasi-Newton relaxation method, LBFGS routine ${ }^{35}$ with its default stop condition.
To estimate the value of the optimal target energy $E$, several preliminary ADMD runs are carried out. The first preliminary run is executed with an overestimated value of $E$. After an ADMD solution is obtained with $E$, successive runs are tried with lower (typically by $1-2 \mathrm{kcal} / \mathrm{mol}$ ) values of $E$. For each successive run, the previous ADMD solution is used as the starting trajectory in an iterative way. The final value of $E$ is set as the smallest, which provides a solution satisfying the total-energy conservation along the folding trajectory. It should be noted that, if the value of $E$ is set too low, ADMD trajectories fail to satisfy the total-energy conservation.

We have carried out 20 independent ADMD calculations where initial pathways are prepared in a random fashion. An initial pathway constitutes a set of successive conformations prepared in real-space, and the difference between two successive conformations is set by using random numbers. When analyzing the ADMD simulation data in the "Results and Discussion" section, to eliminate possible artifacts arising from the choice of an initial pathway, we have extracted common folding features representing the 20 final pathways.

Trajectory for each atom can be represented by a sum of linear interpolation between the initial and final atomic positions and nonlinear variation represented by sine expansion, ${ }^{36}$ as shown below.

$$
\mathbf{q}_{j}=\mathbf{q}_{0}+\left(\mathbf{q}_{P}-\mathbf{q}_{0}\right) \frac{j \Delta}{\tau}+\sum_{k=1}^{P-1} \mathbf{a}_{k} \sin \left(\frac{k \pi j \Delta}{\tau}\right) .
$$

The sine expansion coefficients $\left\{\mathbf{a}_{k}\right\}$ represent details of the nonlinear pathway deviated from the straight line representation. Now, the positions of each atom along the trajectory are represented by $3(P-1)\left\{\mathbf{a}_{k}\right\}$ variables in eq. (6). Finally, eq. (5) is minimized with respect to $3 N(P-1)=3574212$ independent variables. It should be noted that $\left\{\mathbf{a}_{k}\right\}$ provides a natural way to interpolate a pathway, which works well with the multigrid (from $P=20$ to $P=2000$ ) approach used in this work.

The purpose of the ADMD simulation is to find pathways bridging two given states with low potential-energy profile while satisfying the equations of motion. Considering all pathways starting from the given initial structure and arriving at the given final structure following the Newtonian equations of motion, we aim to identify pathways with low potential energy barriers. The potential energy barrier is defined as the potential energy difference between the highest potential energy state and the initial state. Since the entire pathway ensemble satisfying the boundary condition could not be considered, we hope that a total of twenty low potential energy pathways performed in this work, would provide meaningful characteristics of folding mechanism. For each ADMD trajectory, sequential folding event is analyzed in terms of various quantities including the secondary structure element and the overall degree of collapse. Indeed, although details of all twenty ADMD simulations were different from each other, we were able to extract common features of folding free from the randomness of their initial trial pathways. This demonstrates that even a small protein like HP-36 can exhibit a specific folding sequence governed by the energetics of the conformational space. 


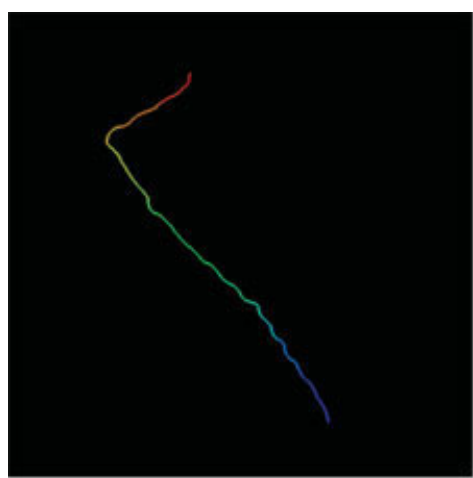

$\mathrm{j}=0$

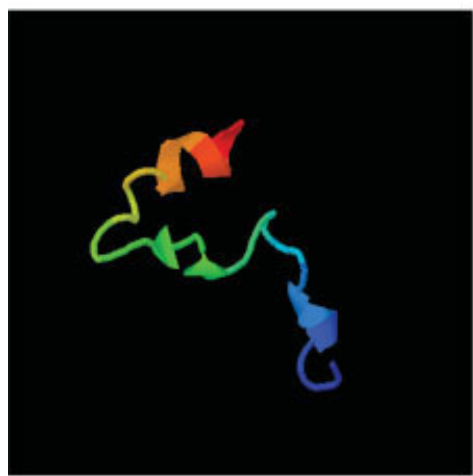

$\mathrm{j}=1000$

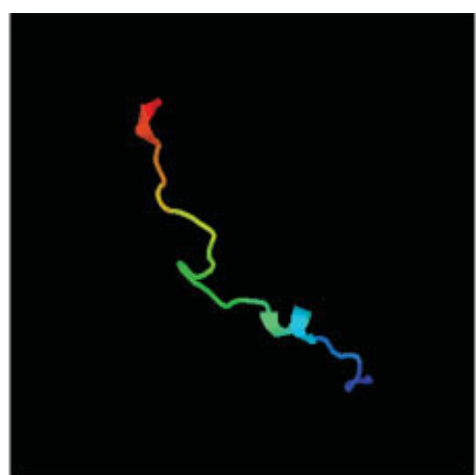

$\mathrm{j}=500$

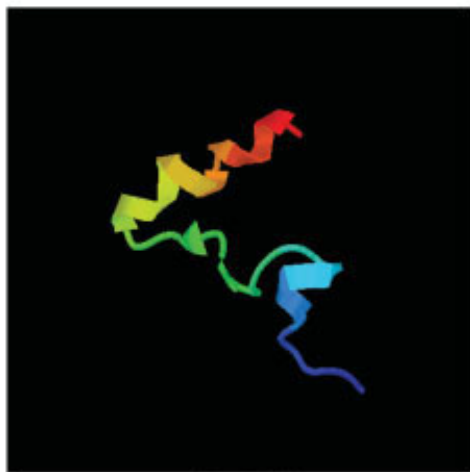

$\mathrm{j}=1300$

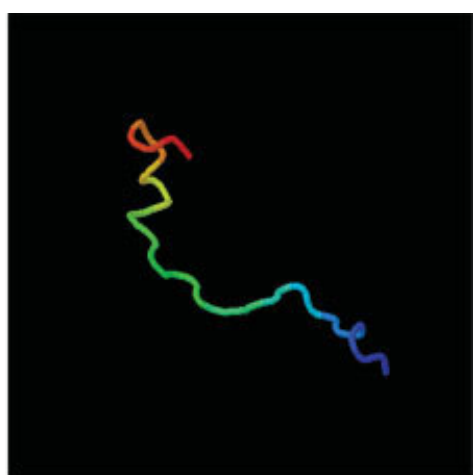

$\mathrm{j}=800$

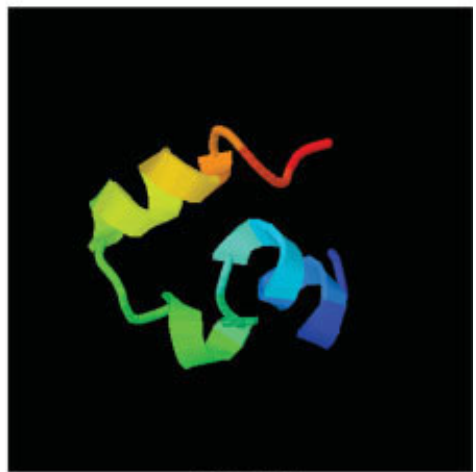

$\mathrm{j}=2000$

Figure 1. Snapshots of the lowest potential-energy-barrier pathway for HP-36 are shown. The initial (final) conformation shown at $j=0(2000)$, is prepared by applying local energy minimization to the fullyextended (NMR native) structure. ADMD simulations are conducted using the AMBER94 all-atom force field with GB/SA solvation model. The variation of colors from blue to red corresponds to the amino-acid sequence of the polypeptide chain from $\mathrm{N}$ - to $\mathrm{C}$-terminus. The snapshots are shown in various scales to fit the whole molecule into the same size picture frame.
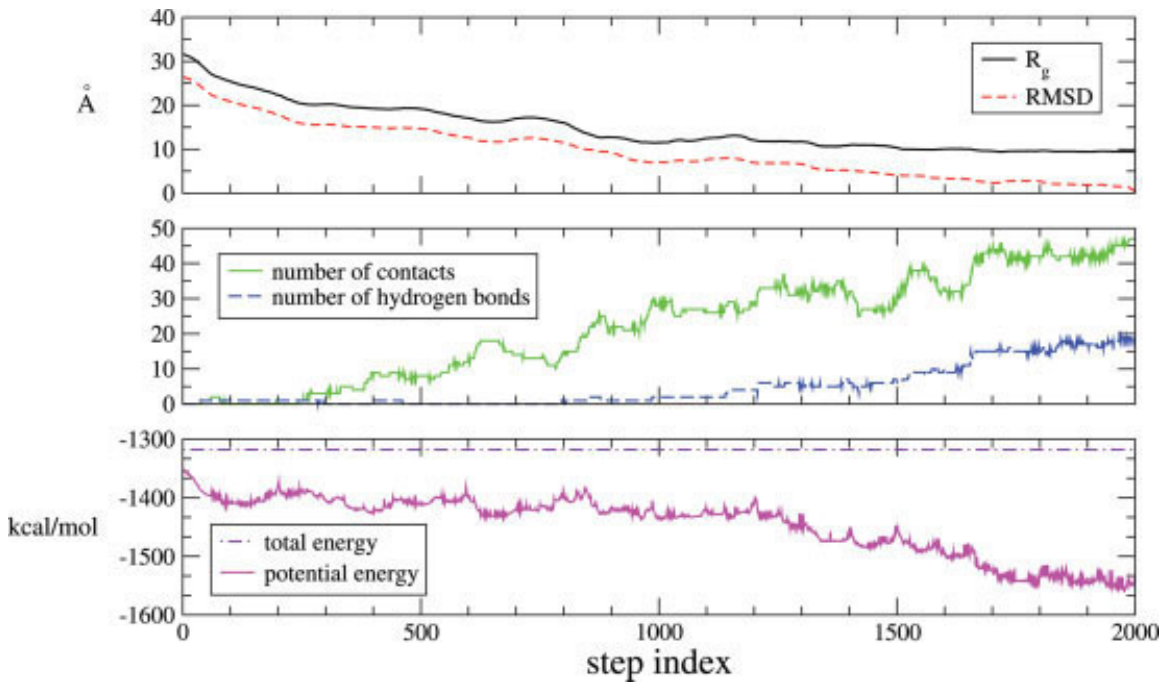

Figure 2. 


\section{Results and Discussion}

\section{Hydrophobic Collapse and Its Specificity}

We have performed 20 independent ADMD simulations, each of which produced a low potential-energy pathway with energy barrier in the range of $3-10 \mathrm{kcal} / \mathrm{mol}$. Initial pathways were prepared in a random fashion, producing variation in pathways. However, these 20 pathways show similar potential-energy fluctuation along their trajectories, and overall folding features independent from initial randomness are considered. We have selected the lowest potentialenergy pathway out of 20 to illustrate the features.

A set of characteristic quantities are shown in Figure 2. Along the pathway, radius of gyration $\left(R_{\mathrm{g}}\right)$ and RMSD from the final native-like conformation are shown in Figure 2. To find the optimal superposition between two structures the quaternion method ${ }^{37,38}$ is used.

We calculated the numbers of native contacts (responsible for the formation of tertiary structure) and native hydrogen bonds (responsible for secondary structure) to quantify the degree of folding process. A native contact is defined to exist between two residues (separated by more than two residues in sequence) if their native $\mathrm{C}^{\alpha}-\mathrm{C}^{\alpha}$ distance is less than $6.5 \AA$. A backbone hydrogen bond is defined to exist between a carbonyl-oxygen and an amide-hydrogen if they are separated by less than $2.5 \AA$ and the virtual bond angle between three atoms (oxygen, nitrogen, and amide-hydrogen) is greater than $135^{\circ}$.

From Figure 2, we observe significant fluctuation in the potential energy whereas the total energy of the system is nicely kept constant. After steep initial decline $(j<250)$, the RMSD decreases roughly at a constant rate throughout the whole folding pathway. In contrast, the overall behavior of the $R_{\mathrm{g}}$ can be divided into two stages separated by $j \sim 950$.

A gradual folding process in terms of the potential-energy variation is observed. During the first folding stage $(j<950), R_{\mathrm{g}}$ decreases drastically from its initial value of $31.6 \AA$ to about 11.8 $\AA$, representing rapid compaction of the protein. During this stage, $R_{\mathrm{g}}$ and RMSD are not sensitive to the detailed fluctuation of the potential energy, and persisting native hydrogen bonds are not yet formed. In the second stage $(j>950)$, the compaction still continues, but at a slower rate. Formation of the native contacts is shown to occur roughly at a constant rate throughout the pathway, which is similar to the overall decrease of RMSD. On the other hand, native hydrogen bonds are formed prominently only in the second folding stage.
At the beginning of the second stage $(j>950)$ only 1 to 2 native hydrogen bonds begin to form, while up to half of the total native contacts are already established. Residues 35 and 36 are observed to move actively toward the residue 8 , and when this happens, the exposed hydrophobic surface of HP-36 is greatly reduced by forming a loosely packed hydrophobic core. The $\mathrm{C}^{\alpha}-\mathrm{C}^{\alpha}$ distance between residues 8 and 35, is rapidly reduced from 68.1 to $12.7 \AA$ A. The collapse occurs along with the partial (40-50\%) tertiary structure formation. These results indicate that, in the folding of HP-36, when 40-50\% tertiary structure is formed, the hydrophobic collapse occurs, and the $\alpha$-helical secondary structure is formed only after the collapse. It is interesting to observe that the potential energy is not much reduced during the initial hydrophobic collapse $(j<950)$. Formation of full native contacts and native hydrogen bonds are associated with the potential-energy improvement during the final stage $(1300 \leq j \leq 2000)$ in a concerted manner.

Now, we turn our attention to the specificity of the hydrophobic collapse. In the conventional hydrophobic collapse model, the collapse is nonspecific. However, through careful examination of all 20 ADMD trajectories, we find that two native contacts between residue pairs of $(8,14)$ and $(21,26)$, are always formed at the collapse. If we denote three helices of the HP-36 as $H 1, H 2$, and $H 3$, their residue numbers are 4-8, 15-18, and 23-30, respectively. Therefore, $(8,14)$ and $(21,26)$ residue pairs are related to the relative orientation between $H 1$ and $H 2$ and the intrahelical interaction of $H 3$.

The $C^{\alpha}$ distances of $(8,14)$ and $(21,26)$ pairs are shown in Figure 3. They settle into their near native values at the end of the first folding stage $(j<950)$. Therefore, contrary to the usual hydrophobic collapse model, the current ADMD simulations suggest that the two specific residue pairs should be near at their native distances upon collapse, which is followed by the concurrent establishment of the secondary structure and full tertiary contacts.

In the literature, ${ }^{22}$ residues $9-32$ are considered as the core residues of HP-36, and in Figure 3, we show the time evolution of the RMSD and $R_{\mathrm{g}}$ of these core residues. We observe that the core RMSD value is reduced drastically twice during $0<j<250$ and $750<j<900$, after which it monotonically vanishes to zero whereas the core $R_{\mathrm{g}}$ stays at its final value.

In summary, in the first folding stage, hydrophobic collapse is observed. Contrary to the conventional hydrophobic collapse model, two specific native contacts are formed upon collapse. After the collapse, the secondary structure and the complete tertiary structure are established concurrently, which will be examined in the "Formation of Native Contacts and Hydrogen Bonds" section.

Figure 2. Various reaction coordinates are shown along the folding pathway for HP-36. They are, in the order shown from top to bottom, the radius of gyration $\left(R_{\mathrm{g}}\right)$, root-mean-square deviation (RMSD) from the final folded structure of NMR, the numbers of native contacts and native hydrogen bonds, the total (kinetic plus potential) energy of the system, and the potential energy. As shown, the total energy conservation is maintained in ADMD simulations. The atomic structures including the first $(j=0)$ and the final $(j=2000)$ states are shown in Figure 1. The pathway can be divided into two stages for convenience. The first stage $(j<950)$ is characterized by the rapid decrease of the protein size $\left(R_{\mathrm{g}}\right)$ and gradual tertiary contact formation with negligible formation of native hydrogen bonds. The $\alpha$-helical secondary structure is formed in the second stage $(j>950)$. In the later steps of the second stage, the potential energy is rapidly improved by the concerted formation of tertiary and secondary structures. In the second stage, the numbers of native hydrogen bonds and native contacts are correlated with correlation coefficient of $r=0.94$. 


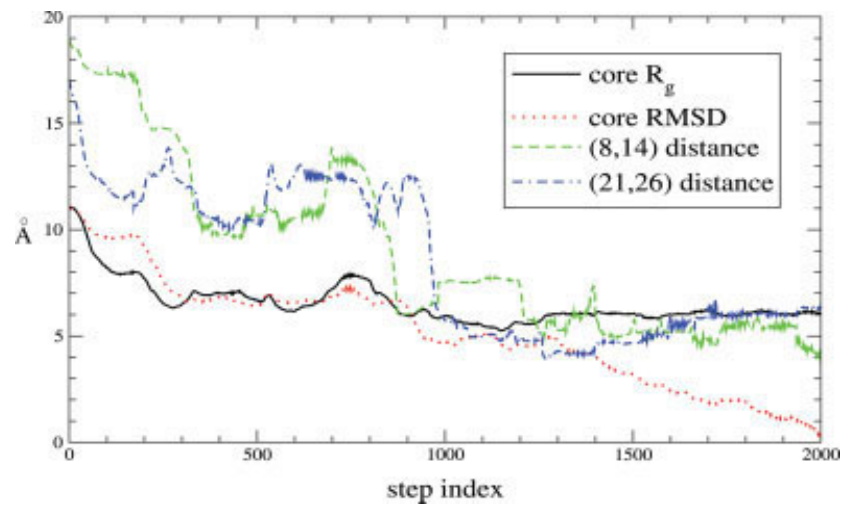

Figure 3. The $C^{\alpha}-C^{\alpha}$ distances between two key residue pairs $(8,14)$ and $(21,26)$ are shown. In all 20 ADMD simulations these two pairs are formed at the collapse. Core RMSD and core $R_{\mathrm{g}}$ measured for residues 9-32 are also shown. The correlation coefficient between the core RMSD and the core $R_{\mathrm{g}}$ is 0.74 . However, the coefficient is 0.85 , if only the first part of the folding process $(0 \leq j \leq 950)$ is considered.

\section{Principal Component Analysis}

Principal component analysis ${ }^{39}$ is used to extract a small number of the most contributing elements (principal components) of the spatial correlation between residue pairs. $M$ defined as

$$
M_{i j}=\left\langle\left(x_{i}-\left\langle x_{i}\right\rangle\right)\left(x_{j}-\left\langle x_{j}\right\rangle\right)\right\rangle,
$$

where $x_{1}, x_{2}, \ldots, x_{3 N_{\alpha}}$ are the Cartesian coordinates of the $N_{\alpha} \mathrm{C}^{\alpha}$ atoms. The average $\langle\ldots\rangle$ is over all structural frames from the ADMD trajectory (i.e., $P+1=2001$ ). By diagonalizing $M, 3 N_{\alpha}$ eigenvectors with their corresponding eigenvalues are obtained.
Figure 4a shows the projection of the ADMD pathway onto the three largest eigenvalue eigenvectors. These three principal components are responsible for a total of $76.4 \%$ structural changes. Significant fluctuation is observed from the first 2 largest principal components for $j<950$, indicating strong collapse. For close examination, the folding trajectory is projected onto the first 2 principal components as shown in Figure 4b. The dominance of the two principal components during the first folding stage $(j<950)$, is in contrast to the second stage $(j>950)$ where their variation is greatly reduced. The first 2 components account for about two thirds of total structural variance. During the final folding stage $(1300<j)$ where fine structural adjustments occur, all three principal components approach to their final values monotonically.

In Figure 5 we show the residue-specific $\mathrm{C}^{\alpha}$ atom contribution to the three principal components. For the first principal component, the $\mathrm{N}$ - and $\mathrm{C}$-terminal parts are moving more than the protein's middle part, whereas for the second component the latter is mainly moving. Residue specificity of the folding is obvious in the figure.

\section{Formation of Native Contacts and Hydrogen Bonds}

Among three helices of HP-36, H3 is the longest with about half of the total helical content. Intrahelical contacts of the three helices are analyzed along the pathway, and the $(i, i+3),(i, i+4)$ contacts together with the number of backbone hydrogen bonds are shown in Figure 6.

In the first folding stage $(j<950)$, transient formation of $(i, i+3),(i, i+4)$ contacts are noticed for $H 1$ and $H 2$. In our earlier ADMD study on the $\alpha$ helix formation of deca-alanine (acetyl(Ala) $10-N$-methyl amide), we found that $(i, i+3)$ contacts precede $(i, i+4)$ contacts. ${ }^{16,40}$ Approximately, the helix formation of HP-36 follows this picture. It is interesting to observe that the simultaneous formation of $(i, i+3)$ and $(i, i+4)$ contacts of $H 1$ for $400<j<700$, results in the breakdown of the $(i, i+4)$ contacts near $j \sim 1000$
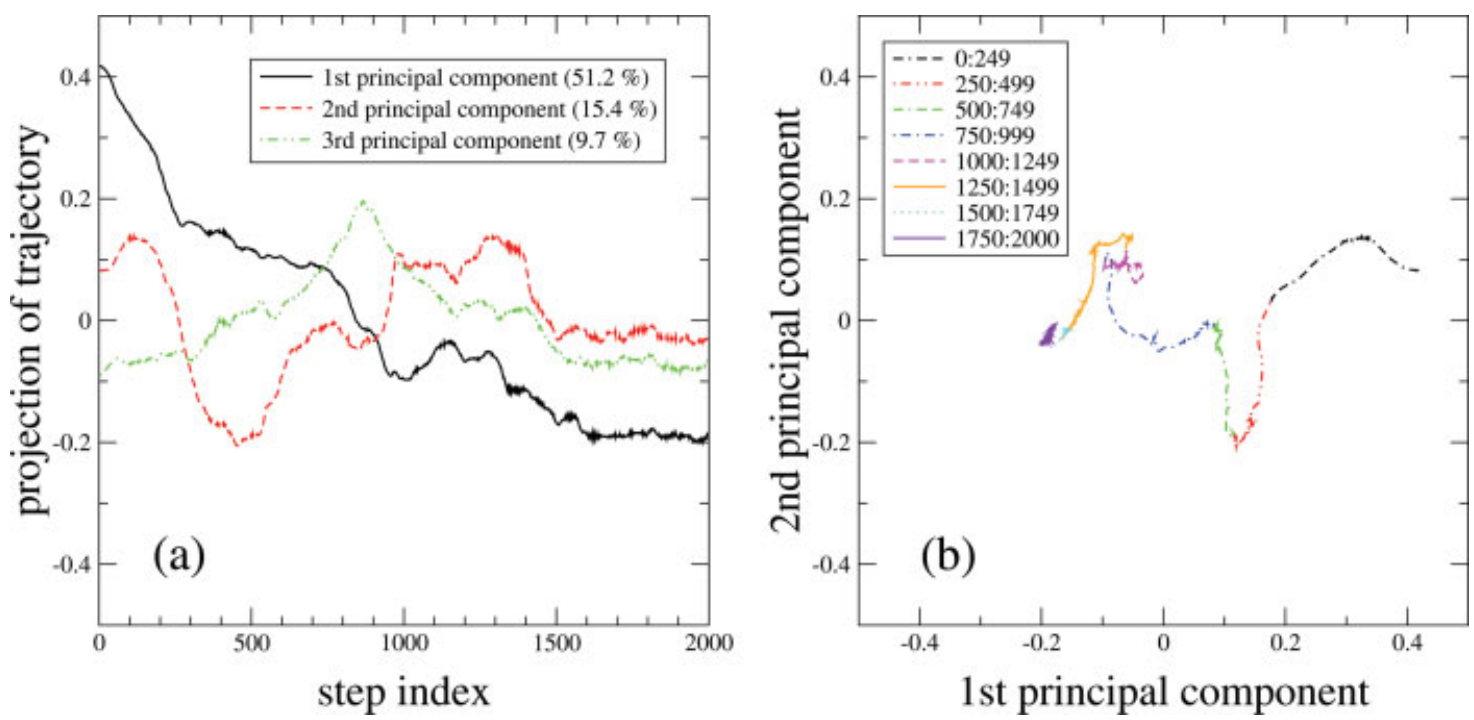

Figure 4. (a) The folding pathway is projected to the first three principal components, which are responsible for a total of $76.4 \%$ of the covariance matrix. (b) The folding trajectory is projected into the first 2 principal components. These two components account for about two thirds of the variance matrix. ADMD pathway steps are indicated by colors. Most movements occur during $j<950$. 


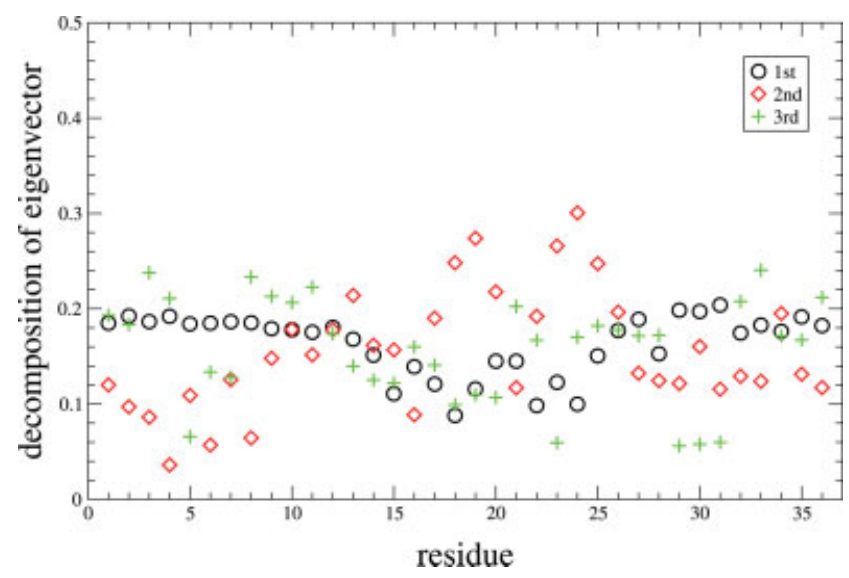

Figure 5. The $\mathrm{C}^{\alpha}$ atom contribution to the first 3 principal components is shown as a function of residue index. The $y$-axis corresponds to the magnitude of three components $(x, y$, and $z)$ contributing to the corresponding principal component. [Color figure can be viewed in the online issue, which is available at www.interscience.wiley.com.]

before regaining them later in the presence of the $(i, i+3)$ contacts. The individual stability of the $(i, i+4)$ contacts in $H 1$ and $H 2$ is not sustained along the pathway as shown in Figure 6. In contrast, this behavior is not observed in $H 3$.

\section{Order of Helix Formation}

The order of helix formation of HP-36 is examined. The initial transient helix formation site varied from simulation to simulation, but mostly it was observed in $H 1$ and $H 3$. Helices are observed to be stabilized mainly by local interaction close in sequence. When we define helix formation by native backbone hydrogen bonds, its order is $H 3, H 1$, and $H 2$. In the recent computational work of Kim et al., ${ }^{41} H 1$ is suggested to be the most stable.

The appearance of sustained backbone hydrogen bonds coincides with the beginning of the second folding stage $(j>950)$. The $\alpha$-helical secondary structures as well as the complete tertiary contacts are established in the second folding stage. Only after $H 3$ and $H 1$ are established in their native relative orientation, $H 2$ is formed.

We note that the helix formation order of HP-36 from this study is a bit different from that of HP-35 by Lei and Duan ${ }^{24}$ where $H 1$ is formed last. They ${ }^{24}$ suggested that the folding of $H 1$ requires a prefolded segment ( $H 2$ and $H 3$ ), representing the cooperativity of protein folding. Their observation that $H 1$ forms after $H 3$ is consistent with this study. On the other hand, Ensign et al. ${ }^{25}$ showed that the fast folding of the HP-35_NleNle where two lysines at the 24th and 29th positions are replaced by norleucines is due to the stable formation of $H 1$ and hydrophobic core in the early unfolded state. In all 20 ADMD simulations, the first sustained native hydrogen bond is established always in $H 3$. However, after this, we observe variation in the sequence of native contact formation. Because of the topological reason, the formation of native contacts is closely tied to the formation of native hydrogen bonds. Nevertheless, the sequence of native contact formation varied among 20 ADMD trajectories, representing the existence of multiple folding pathways.

\section{Final Folding Stage}

We now turn our attention to the final folding stage of HP-36. Examination of Figures 2, 3, and 6, for $j>1300$ can be summarized as follows. The core $R_{\mathrm{g}}$ and $(i, i+3)$ contacts are already near their final values at $j=1300$, and the core RMSD decreases to 0 at a constant rate. The helix contents represented by the number of backbone hydrogen bonds and $(i, i+4)$ contacts increase in a steady fashion while the potential energy improves rapidly. Good correlations between the core RMSD, the helix contents, and the potential energy are observed with the average correlation of 0.9 . This indicates that, at the final folding stage, fine assembly of secondary structures occurs and the potential energy improves steadily due to the gradual increase of both the number of native contacts and the number of native hydrogen bonds, representing the cooperativity of helix formation.

\section{Discussion on Folding Mechanism}

The folding pathway of HP-36 suggested by current ADMD simulations is in contrast with the framework model, ${ }^{42}$ where secondary structures form first and then the tertiary structure is organized by packing the already established secondary elements. We find that secondary structure begins to form only after a loose hydrophobic core with some tertiary contacts is established. The collapse is observed by the rapid reduction of protein size in the first stage of folding. This observation that the secondary structure is formed in the presence of partial tertiary structure, is consistent with experimental observation. ${ }^{43}$

ADMD pathway for HP-36 is consistent with the hydrophobic collapse model ${ }^{44}$ in that the tertiary and secondary structures are formed concurrently after the initial hydrophobic collapse. After the collapse (i.e., $j>950$ ), we find a good correlation between the number of native hydrogen bonds (secondary structure) and the number
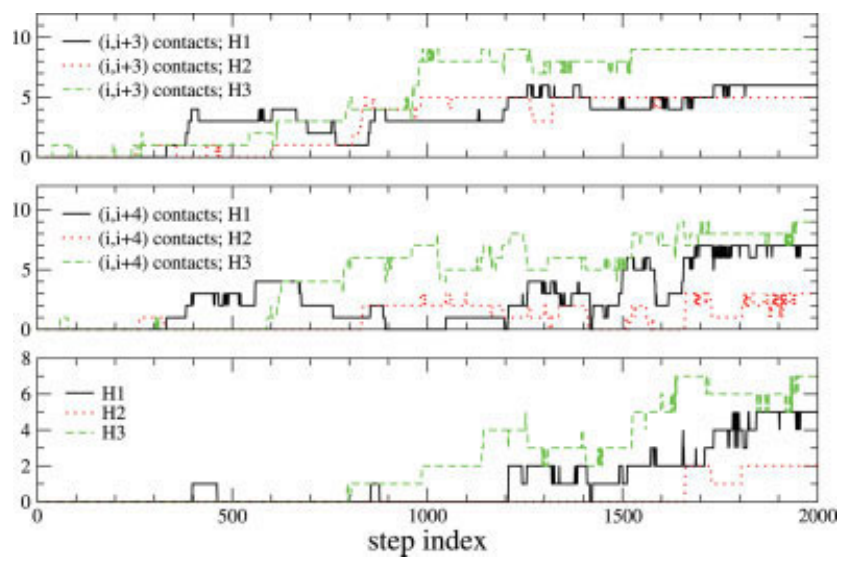

Figure 6. The numbers of native contacts and native hydrogen bonds are shown along the folding pathway for three helices, $H 1, H 2$, and $H 3$. The order of helix formation is $H 3, H 1$, and $H 2$. Overall, we observe that $(i, i+3)$ contacts are followed by $(i, i+4)$ contacts for surviving helices. Although initial transient helix formation is recorded from $H 1$, $H 3$ is always the first to form. [Color figure can be viewed in the online issue, which is available at www.interscience.wiley.com.] 
of native contacts (tertiary structure), with the correlation coefficient of 0.94. Thus, as far as the correlation between the formation of the secondary structures and the completion of the tertiary structure is concerned the present folding model is consistent with both computational $^{3,4}$ and experimental ${ }^{43}$ results. The current study also agrees with the computational work by Duan et al., ${ }^{3}$ where it is shown that the burst phase precedes the slow conformational readjustment phase in HP-36 folding.

In this study, initial hydrophobic collapse is shown to be associated with a couple of specific native contacts contrary to the conventional hydrophobic collapse model, where the nonspecificity is a key feature. These specific nonlocal contacts are shown to reduce $R_{\mathrm{g}}$. A recent computational model ${ }^{12}$ also suggests that formation of significant protein structure occurs only after the formation of a specific tertiary structure. Another folding study of HP-36 based on worldwide-distributed computing ${ }^{10}$ also suggested the hydrophobic collapse as the folding mechanism. However, nonspecific collapse was suggested with a fast relaxation.

In the diffusion-collision model, ${ }^{45}$ the intrinsic stability of helices and nonlocal interactions drive the formation of secondary structure. However, the fact that only about $10 \%$ of native hydrogen bonds are observed in this study at the end of the hydrophobic collapse, suggests that HP-36 does not follow the diffusion-collision model where a significant amount of helix formation $(>50 \%)$ before collapse is suggested. This is probably due to the low intrinsic helical stability/propensity of the three helices in HP-36 when exposed in water. ${ }^{43,46}$

One may want to identify the transition state along the ADMD folding pathway obtained in this work, for which free energy profile projected on a well-defined reaction coordinate should be calculated. Although the ADMD formalism does not provide direct ways to obtain free energy profiles, one may consider to perform Monte Carlo/molecular dynamics simulations to estimate the free energy difference between successive conformations along the pathway, which is beyond the scope of this work.

\section{Conclusions}

We have applied the action-derived molecular dynamics (ADMD) to investigate the folding dynamics of HP-36, a small three-helix protein. In the first stage of the folding pathway, overall size of the protein measured by $R_{\mathrm{g}}$ was drastically reduced, representing rapid compaction of the protein. A loose hydrophobic core is shown to be formed at the end of the first folding stage. The calculated folding pathway is similar to the hydrophobic collapse model, ${ }^{44}$ in which the concurrent formation of tertiary structure (native contacts) and secondary structure ( $\alpha$-helical content) is emphasized on an equal footing after hydrophobic collapse. Stable secondary structures are formed only after the collapse, suggesting that HP-36 folding follows neither the framework model nor the diffusion-collision model.

After the initial hydrophobic collapse, $H 3$ the longest of the three helices begins to form, which is followed by $H 1$ positioned in the native orientation to $H 3$. $H 2$ is shown to form at the final folding stage. In all 20 ADMD simulations, the first lasting intrahelical backbone hydrogen bond was always observed in $H 3$. However, we observed variation in the subsequent tertiary contact formation among ADMD pathways, indicating the existence of multiple folding pathways.

Finally, a description of the folding pathway in terms of principal component analysis is presented to characterize the folding dynamics in reduced dimensions. With only three principal components, we were able to describe $76.4 \%$ of the pathway.

\section{References}

1. Dobson, C. M. Nature (London) 2003, 426, 884.

2. Jones, C. M.; Henry, E. R.; Hu, Y.; Chan C. K; Luck S. D.; Bhuyan, A.; Roder, H.; Hofrichter, J.; Eaton, W. A. Proc Natl Acad Sci USA 1993, 90, 11860.

3. Duan, Y.; Kollman, P. A. Science 1998, 282, 740.

4. Duan, Y.; Wang, L; Kollman, P. A. Proc Natl Acad Sci USA 1998, 95, 9897.

5. Brooks, C. L. III; Gruebele, M.; Onuchic, J. N.; Wolynes, P. G. Proc Natl Acad Sci USA 1998, 95, 11037.

6. Guo, Z.; Brooks, C. L. III; Boczko, E. M. Proc Natl Acad Sci USA 1997, 94, 10161.

7. Lazaridis, T.; Karplus, M. Science 1997, 278, 1928.

8. Snow, C. D.; Nguyen, H.; Pande, V. S.; Gruebele, M. Nature (London) 2002, 420, 102.

9. Zhou, Y.; Karplus, M. Nature (London) 1999, 401, 400.

10. Zagrovic, B.; Snow, C. D.; Shirts, M. R.; Pande, V. S. J Mol Biol 2002, 323, 927.

11. Jang, S.; Kim, E.; Shin, S.; Pak, Y. J Am Chem Soc 2003, 125, 14841.

12. Wickstrom, L.; Okur, A.; Song, K.; Hornak, V.; Raleigh, D. P.; Simmerling, C. J Mol Biol 2006, 360, 1094

13. Allen M. P.; Tildesley, D. J. Computer Simulation of Liquids; Oxford University Press: New York, 1987.

14. Passerone, D; Parrinello, M. Phys Rev Lett 2001, 87, 108302.

15. Lee, I.-H.; Lee, J.; Lee, S. Phys Rev B 2003, 68, 064303.

16. Lee, I.-H.; Kim, S.-Y.; Lee, J. Chem Phys Lett 2005, 412, 307.

17. McKnight, C. J.; Matsudaira, P. T.; Kim, P. S. Nat Struc Biol 1997, 4, 180

18. Wang, M.; Tang, Y.; Sato, S.; Vugmeyster, L.; McKnight, C. J.; Raleigh, D. P. J Am Chem Soc 2003, 125, 6032

19. Kubelka, J.; Eaton, W. A.; Hofrichter, J. J Mol Biol 2003, 329, 625.

20. Chiu, T. K.; Kubelka, J.; Herbst-Irmer, R.; Eaton, W. A.; Hofrichter, J.; Davies, D. R. Proc Natl Acad Sci USA 2005, 102, 7517.

21. Kubelka, J.; Chiu, T. K.; Davies, D. R.; Eaton, W. A.; Hofrichter, J. J Mol Biol 2006, 359, 546.

22. De Mori, G. M. S.; Colombo, G.; Micheletti, C. Proteins 2005, 58, 459.

23. Brewer, S. H.; Vu, D. M.; Tang, Y.; Li, Y.; Franzen, S.; Raleigh, D. P.; Dyer, R. B. Proc Natl Acad Sci USA 2005, 102, 16662.

24. Lei, H.; Duan, Y. J Mol Biol 2007, 370, 196.

25. Ensign, D. L.; Kasson, P. M.; Pande, V. S. J Mol Biol 2007, 374, 806.

26. Elber, R.; Cárdenas, A.; Ghosh, A.; Stern, H. A. Adv Chem Phys 2003, 126, 93

27. Verlet, L. Phys Rev 1967, 159, 98

28. Cornell, W. D.; Cieplak, P.; Bayly, C. I.; Gould, I. R.; Merz, K. M., Jr.; Ferguson, D. M.; Spellmeyer, D. C.; Fox, T.; Caldwell, J. W.; Kollman, P. A. J Am Chem Soc 1995, 117, 5179.

29. Qiu, D.; Shenkin, P. S.; Hollinger, F. P.; Still, W. C. J Phys Chem A 1997, 101, 3005.

30. Ponder, J. W.; Richard, F. M. J Comput Chem 1987, 8, 1016: http://dasher.wustl.edu/tinker

31. Kirkpatric, S.; Gelatt, C. D., Jr.; Vecci M. P. Science 1983, 220, 671. 
32. Lee, J.; Scheraga, H. A.; Rackovsky, S. J Comput Chem 1997, 18, 1222.

33. Lee, J.; Lee, I.-H.; Lee, J. Phys Rev Lett 2003, 91, 080201.

34. Lee, I.-H.; Kim, Y.-H.; Martin, R. M. Phys Rev B 2000, 61, 4397.

35. Liu, D. C.; Nocedal, J. Math Program B 1989, 45, 503.

36. Cho, A. E.; Doll, J. D.; Freeman, D. L. Chem. Phys. Lett. 1994, 229, 218.

37. Kearsley, S. J. J Comput Chem 1990, 11, 1187.

38. Coutsias, E. A.; Seok, C.; Dill, K. A. J Comput Chem 2004, 25, 1849.

39. García, A. E. Phys Rev Lett 1992, 68, 2696.
40. Millhauser, G. L.; Stenland, C. J.; Hanson, P.; Bolin, K. A.; Vandeven, F. J. M. J Mol Biol 1997, 267, 963.

41. Kim, S.-Y.; Lee, J.; Lee, J. J Chem Phys 2004, 120, 8271.

42. Kim, P. S.; Baldwin, R. L. Annu Rev Biochem 1990, 59, 631.

43. Reymond, M. T.; Merutka, G.; Dyson, H. J.; Wright, P. E. Protein Sci 1997, 6, 706.

44. Ptitsyn, O. B. J Protein Chem 1987, 6, 273.

45. Karplus, M.; Weaver, D. L. Nature (London) 1976, 260, 404.

46. Fernández, A.; Shen, M.-Y.; Colubri, A.; Sosnick, T. R.; Berry, R. S.; Freed, K. F. Biochemistry 2003, 42, 664. 